\title{
BMJ Open Contribution of lung ultrasound in diagnosis of community-acquired pneumonia in the emergency department: a prospective multicentre study
}

\author{
François Javaudin, ${ }^{1,2}$ Nicolas Marjanovic, ${ }^{3}$ Hugo de Carvalho (D) ,2 \\ Benjamin Gaborit, ${ }^{4}$ Quentin Le Bastard, ${ }^{2}$ Estelle Boucher, ${ }^{5}$ Denis Haroche, ${ }^{6}$ \\ Emmanuel Montassier (D) , ${ }^{1}$ Philippe Le Conte (D) ${ }^{1,2}$
}

To cite: Javaudin $F$, Marjanovic N, de Carvalho H, et al. Contribution of lung ultrasound in diagnosis of community-acquired pneumonia in the emergency department: a prospective multicentre study. BMJ Open 2021;11:e046849. doi:10.1136/ bmjopen-2020-046849

- Prepublication history for this paper is available online. To view these files, please visit the journal online (http://dx.doi. org/10.1136/bmjopen-2020046849).

Received 10 November 2020 Accepted 28 July 2021

Check for updates

(c) Author(s) (or their employer(s)) 2021. Re-use permitted under CC BY-NC. No commercial re-use. See rights and permissions. Published by BMJ.

For numbered affiliations see end of article.

Correspondence to

Professor Philippe Le Conte; philippe.leconte@chu-nantes.fr

\section{ABSTRACT}

Lung ultrasound (LUS) can help clinicians make a timely diagnosis of community-acquired pneumonia (CAP).

Objectives To assess if LUS can improve diagnosis and antibiotic initiation in emergency department (ED) patients with suspected CAP.

Design A prospective observational study.

Settings Four EDs.

Participants The study included 150 patients older than 18 years with a clinical suspicion of CAP, of which 2 were subsequently excluded (incorrect identification), leaving 148 patients ( 70 women and 78 men, average age $72 \pm 18$ years). Exclusion criteria included a life-threatening condition with do-not-resuscitate-order or patient requiring immediate intensive care.

Interventions After routine diagnostic procedure (clinical, radiological and laboratory tests), the attending emergency physician established a clinical CAP probability according to a four-level Likert scale (definite, probable, possible and excluded). An LUS was then performed, and another CAP probability was established based on the ultrasound result. An adjudication committee composed of three independent experts established the final CAP probability at hospital discharge.

Primary and secondary outcome measures Primary objective was to assess concordance rate of CAP diagnostic probabilities between routine diagnosis procedure or LUS and the final probability of the adjudication committee. Secondary objectives were to assess changes in CAP probability induced by LUS, and changes in antibiotic treatment initiation.

Results Overall, $27 \%$ (95\% Cl 20 to 35) of the routine procedure CAP classifications and $77 \%$ (95\% $\mathrm{Cl} 71$ to 84$)$ of the LUS CAP classifications were concordant with the adjudication committee classifications. Cohen's kappa coefficients between routine diagnosis procedure and LUS, according to adjudication committee, were 0.07 (95\% Cl 0.04 to 0.11 ) and 0.61 (95\% $\mathrm{Cl} 0.55$ to 0.66$)$, respectively. The modified probabilities for the diagnosis of CAP after LUS resulted in changes in antibiotic prescriptions in $32 \%$ (95\% Cl 25 to 40$)$ of the cases.
Strengths and limitations of this study

- This is a multicentre pragmatic study, including adults with presumptive diagnosis of communityacquired pneumonia in emergency departments.

- Lung ultrasounds (LUSs) were performed according to a defined protocol by investigators with a wide range of experience, from beginners to experts.

- Investigation tools (chest X-ray and laboratory tests) were conducted at the physician's discretion; this fact increases the external validity of the study.

- The inclusion of a convenience sample of patients may have induced a selection bias.

- The adjudication committee had access to LUS raw data, which may have influenced their diagnosis.

Conclusion In our study, LUS was a powerful tool to improve CAP diagnosis in the ED, reducing diagnostic uncertainty from $73 \%$ to $14 \%$.

Trial registration number NCT03411824.

\section{INTRODUCTION}

Community-acquired pneumonia (CAP) is a common cause of acute infection worldwide and is responsible for frequent hospital admissions. ${ }^{1}$ CAP remains the leading infectious disease cause of death in the USA. ${ }^{2}$ Early antibiotic treatment is crucial to reduce in-hospital mortality, ${ }^{3}$ emphasising the need for a timely detection and diagnosis. However, antibiotic overuse, mainly in lower respiratory tract infections, is a critical public health concern leading to increased bacterial resistance. ${ }^{4}$ Meanwhile, the diagnosis of CAP in the emergency department (ED) is difficult given the limitations of clinical examination, chest X-ray (CXR) and laboratory tests. Indeed, association of cough, fever, tachycardia and crackles provides a CAP probability between 
$20 \%$ and $50 \% .^{5}$ Thus, the aetiologic diagnosis of an acute shortness of breath in the ED remains challenging, especially in elderly patients or those with concomitant chronic cardiac or pulmonary diseases. ${ }^{5}$ In this population, diagnostic accuracy is difficult to achieve and the need for hospitalisation or the risk of death are higher than in younger patients. ${ }^{7}$ The usual diagnostic workup, that is, without LUS or CT scan, can overlook mild pneumonia, delaying appropriate treatment, which can lead to an increased mortality risk. ${ }^{89}$

While CXR is the most commonly used imaging modality to access CAP diagnosis, a significant inter observer variability has been demonstrated. ${ }^{10}$ Furthermore, the intrinsic qualities of CXR for CAP diagnosis are limited, with a sensitivity and specificity of $43 \%$ and $93 \%$, respectively. ${ }^{11}$ A recent prospective multicentre study in patients with suspected CAP assessed the diagnosis changes induced by a CT scan of the chest. ${ }^{12}$ The study found that CT scan improves diagnosis and management of ED patients with suspected CAP in terms of antibiotic initiation and hospitalisation. However, CT scan of the chest for CAP diagnosis is not routinely feasible in many ED and would expose to significant radiation. ${ }^{13}$

Lung ultrasound (LUS) is increasingly used in routine practice and can help the clinician make a rapid diagnosis in patients with acute respiratory failure. ${ }^{14}{ }^{15} \mathrm{~A}$ meta-analysis showed that LUS sensitivity ranged from $80 \%$ to $90 \%$ and specificity from $70 \%$ to $90 \%{ }^{16}$ Similar results were found in ED focused meta-analysis with pooled sensitivity of $92 \%$ and pooled specificity of $93 \%{ }^{17}$ Moreover, LUS sensitivity was found better than CXR in a prospective study. ${ }^{18}$ As CT scan changed the diagnosis probability in patients with suspected pneumonia, ${ }^{12}$ the rationale for our study was to assess the role of LUS, an easier accessible imaging modality, in suspected adults with CAP visiting the ED. As ED physicians play a major role in the initial work-up of CAP, we aim to investigate the diagnosis performance of LUS for suspected CAP and its impact on antibiotic treatment initiation in the ED. ${ }^{19}$

\section{Patients and methods}

Study design and setting

EchoPAC was a prospective study in four EDs between November 2016 and December 2018.

\section{Selection of participants}

The patients of the present study were a convenience sample of patients aged more than 18 years for whom the attending emergency physician made the presumptive diagnosis of CAP, based on signs and symptoms such as new onset of fever, pleural pain, shortness of breath, purulent expectoration and localised or bilateral auscultation abnormalities. ${ }^{19}$ Patients were enrolled when a local investigator was available. Standard laboratory and radiographic testing were conducted at the discretion of the patient's treating clinician. The CXR was interpreted by the attending emergency physician. We excluded patients with a life-threatening condition with do-not-resuscitate-order, patient requiring immediate intensive care or with known pregnancy. Patients requiring immediate intensive care were excluded as (a) they could not consent to the study, (b) priority was given to placing the patient on mechanical ventilation and (c) these patients often had a CT scan before the LUS could be performed by investigators.

\section{Study intervention}

Informed consent approval was obtained and routine diagnosis procedure was performed by the treating emergency physician. This procedure included clinical, radiological and laboratory tests. X-ray was performed preferably with the patient in standing position with posteroanterior ray. When it was not possible for the patient to stand up, the chest X-ray was performed in a sitting position with anteroposterior ray. Once the routine diagnostic procedure was fulfilled, the patient's treating emergency physician established: (a) a CAP probability using a four-level Likert scale (definite, probable, possible and excluded) and (b) the need for antibiotic treatment. As in the Claessens et al's study, ${ }^{12}$ this classification was not a validated diagnosis classification but a global impression on CAP diagnosis. Once the standard procedure for CAP diagnosis was performed by the treating emergency physician, who established a CAP probability and proposed a treatment plan accordingly, an LUS was then performed by a local investigator blind to the antibiotic treatment. After the ultrasonography assessment, an LUS performer established another probability for CAP diagnosis and treatment (post LUS probability).

LUS was performed as soon as possible after the routine diagnosis procedure, using a 12-point method, and with a 3.5-5 MHz curved array probe. The operator was aware of the presumptive diagnosis of CAP. There were six scanning points on each hemithorax (figure 1): two anterior (up and down, between the sternum and the anterior

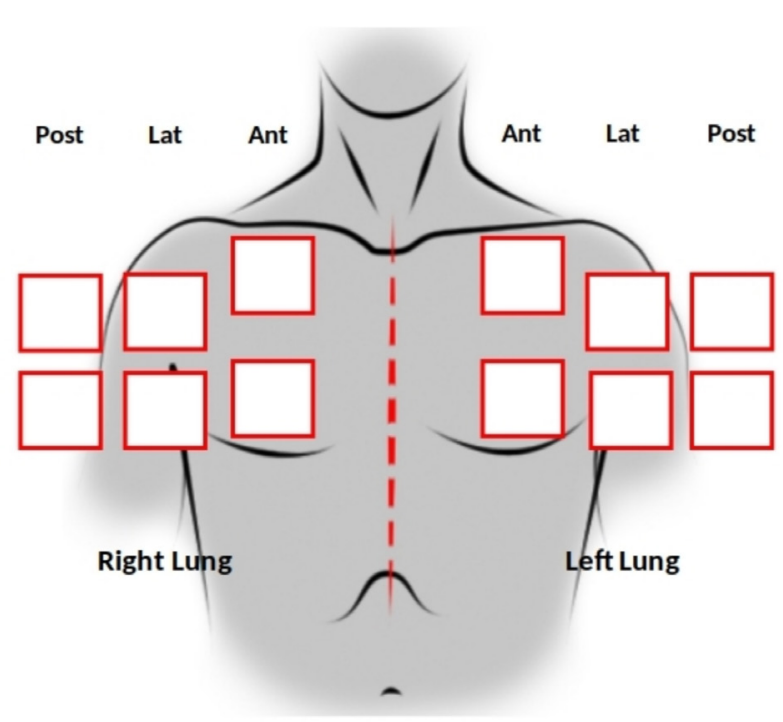

Figure 1 Lung ultrasound procedure applied in the echoPAC study. 
on their expert analysis of the data described above) using the same four-level Likert scale, which was used for the standard diagnosis.

\section{Objectives}

The primary objective was to assess the impact of LUS on diagnostic accuracy of CAP, by measuring the concordance rate of CAP diagnostic probabilities between routine diagnosis procedure or LUS and the final probability of the adjudication committee. The secondary objectives were to assess the changes in CAP probability induced by LUS, the changes in antibiotic treatment initiation, the self-reported experience (beginner, experienced and expert) and the duration and difficulty (from 1 impossible to 10 very easy) of LUS.

\section{Statistical analyses and sample size calculation}

Statistical analyses were performed using PASW Statistics (SPSS, released 2009, PASW Statistics for Windows, V.18.0). Continuous data were expressed as mean and SD if normally distributed, or median and IQR. Categorical data were reported as percentages and $95 \%$ CI. Continuous data were compared using Wilcoxon test, categorical data with $\chi^{2}$ test or the McNemar's test. Diagnosis concordances were explored using the Cohen's kappa coefficient. A p value $<0.05$ was considered significant. Based on a previous monocentric study (unpublished data), we anticipated a concordance rate with the adjudication committee probability of $55 \%$ for the clinical probability and $80 \%$ for the post LUS probability. With a type I error of 0.05 and a $90 \%$ power to detect this difference, the required number of patients would be 144 . or impossibility to conclude. In the context of presumptive diagnosis of CAP, the LUS findings confirming the diagnosis were a localised consolidation with or without surrounding effusion or a unilateral presence of more than three B lines. ${ }^{141520}$ To comply with protocol, investigators were not allowed to use high frequency linear array probe. The operator reported his experience (beginner, experienced and expert), the LUS difficulty (from 1 impossible to 10 very easy) and the duration. All operators attended various training courses and were classified as follows: beginners for less than 30 exams performed, experienced for 30-100 exams performed and expert for at least 100 exams performed.

\section{Adjudication committee}

An adjudication committee was established, involving three independent experts in emergency medicine, internal medicine and infectious diseases. They independently reviewed all data collected from case report forms, including routine diagnosis procedure (clinical, radiological and laboratory tests), LUS raw data, patient follow-up during hospital stay and the discharge diagnosis. They were blinded to each other's review and were not aware of both clinical and LUS CAP probabilities. When the experts were not unanimous, the final result was determined after discussion. For each patient, the adjudication committee established a probability (based

\section{RESULTS}

\section{Characteristics of study subjects and routine diagnosis procedure}

During the study period, 150 patients were included and 2 were excluded due to incorrect identification, leaving 148 analysed patients (figure 2). The main characteristics are indicated in table 1 . The mean age was $72 \pm 18$ years (range: $19-100$ years), 71 patients $(48 \%)$ were aged more than 75 years and $52 \%$ were men. Routine diagnosis procedure involved laboratory tests in 135 patients, and CXR in 138 patients. Following routine diagnosis procedure, CAP probabilities were assessed as follows: definite: $34(23 \%)$, probable: $52(35 \%)$, possible: $56(38 \%)$ and excluded: $6(4 \%)$. Initiation of antibiotic treatment was decided for 106 patients (72\%).

\section{Impact of LUS on CAP management in the ED}

LUS examination results modified CAP probability classification in 106 patients, $72 \%$ (95\% CI 61 to 80 ), as detailed in table 2, and 82 modifications. After LUS, CAP was classified as definite in 97 patients $(66 \%)$, probable in $13(9 \%)$, possible in $8(5 \%)$ and excluded in $30(20 \%)$ (table 3). The most frequent changes affected probable or possible categories: 108 patients from these categories 
Table 1 Baseline characteristics of study patients

\begin{tabular}{ll}
\hline Characteristics & $\begin{array}{l}\text { No. (\%) or } \\
\text { mean } \pm \text { SD }\end{array}$ \\
\hline Age (years) & $72 \pm 18$ \\
\hline Sex (male) & $78(53)$ \\
\hline Temperature $\left({ }^{\circ} \mathrm{C}\right)$ & $37.6 \pm 1.1$ \\
\hline Heart rate (beats/min) & $96 \pm 20$ \\
\hline Respiratory rate (movements/min) & $26 \pm 6$ \\
\hline Systolic arterial pressure $(\mathrm{mm} \mathrm{Hg})$ & $124 \pm 27$ \\
\hline Diastolic arterial pressure $(\mathrm{mm} \mathrm{Hg})$ & $68 \pm 20$ \\
\hline Oxygen saturation $(\%)$ & $95 \pm 4$ \\
\hline Crackles & $66(45)$ \\
\hline Chest X-ray & \\
\hline Parenchymal infiltrate & $131(88)$ \\
\hline Including unilateral finding & $69(53)$ \\
\hline Including bilateral finding & $62(47)$ \\
\hline Pleural effusion & $28(19)$ \\
\hline
\end{tabular}

after routine diagnosis procedure were reclassified as definitive $(n=64,59 \%)$ and excluded $(n=27,25 \%)$, leaving 21 patients $(14 \%)$ in probable or possible categories following LUS (figure 3). After LUS, other diagnoses were chronic obstructive pulmonary disease exacerbation $(\mathrm{n}=11,7 \%)$, acute heart failure $(\mathrm{n}=11,7 \%)$, other or no diagnosis $(n=14,9 \%)$.

Following LUS, 47 changes (32\%; 95\% CI 25 to 40), in antibiotic prescriptions, were proposed: 21 antibiotic treatments $(45 \% ; 95 \%$ CI 31 to 59$)$ were started, while 26 (55\%; 95\% CI 41 to 69) were discontinued. Adequacy between antibiotics prescription and final diagnosis in these 47 patients is displayed in table 3 .

\section{Adjudication committee CAP probability}

The adjudication committee was initially unanimous in 137 patients and an agreement was reached after discussion in the remaining 11 patients. Adjudication committee CAP probability was definite in 81 patients $(55 \%)$, probable in $16(11 \%)$, possible in $12(8 \%)$ and excluded in 39 (26\%) (table 2). Overall, compared with the adjudication committee CAP probability, 39 out of 148 routine diagnosis procedure CAP probability were correct, while 109 LUS CAP probability were correct, $27 \%$ (95\% CI 20 to 35 ) versus $77 \%$ (95\% CI 71 to 84), respectively; $\mathrm{p}<0.001$ (figure 3). Cohen's kappa coefficients between routine diagnosis procedure and LUS, according to the adjudication committee, were 0.07 (95\% CI 0.04 to 0.11 ) and 0.61 (95\% CI 0.55 to 0.66$)$, respectively.

\section{Feasibility of LUS in the ED}

There were 13 operators with self-reported experiences as follows: beginner for 4, experienced for 4 and expert for 5. Impossibility to acquire the images was reported for 55 scanning points $(6 \%)$ in 20 patients. Self-reported difficulty of LUS was $8.1 \pm 2.2$ (ie, easy) and mean duration of LUS was $6 \pm 2.4 \mathrm{~min}$.

\section{DISCUSSION}

In our prospective study, including ED patients with presumptive diagnosis of CAP, we found that LUS modified the probability of CAP diagnosis in $72 \%$ of the cases, mostly $(77 \%)$ in agreement with the probability of the adjudication committee. The major finding was that LUS reduced diagnostic uncertainty from $73 \%$ to $14 \%$. We also observed that LUS findings resulted in changes in antibiotic prescriptions in $30 \%$ of the patients. Using a different primary endpoint (change of CAP probability), a prospective study reported high-accuracy diagnosis of LUS in patients with suspected CAP. ${ }^{19}$ Indeed, LUS has been recognised for several years to have a very good diagnostic performance in CAP with a positive likelihood ratio (LR) of 16.8 and a negative LR of 0.07 (meta-analysis, including 10 studies dating from 1996 to 2013). ${ }^{21}$ Our results are in line with these previous studies, which show

Table 2 Distribution of changes in CAP probability classification before and after LUS in 148 patients

\begin{tabular}{|c|c|c|c|c|c|c|c|}
\hline & \multicolumn{4}{|c|}{ LUS CAP probability } & \multirow[b]{2}{*}{ Total } & \multicolumn{2}{|c|}{ Changes in classifications } \\
\hline & Definite & Probable & Possible & Excluded & & Number & $\begin{array}{l}\text { Rates } \\
(95 \% \mathrm{Cl})\end{array}$ \\
\hline \multicolumn{8}{|c|}{ Routine diagnosis procedure CAP probability } \\
\hline Definite & 31 & 1 & 0 & 2 & $34(23 \%)$ & 3 & $9 \%$ (3 to 24$)$ \\
\hline Probable & 30 & 5 & 3 & 14 & $52(35 \%)$ & 47 & $90 \%$ (79 to 96$)$ \\
\hline Possible & 34 & 4 & 5 & 13 & $56(38 \%)$ & 51 & $91 \%$ (80 to 96$)$ \\
\hline Excluded & 2 & 3 & 0 & 1 & $6(4 \%)$ & 5 & $83 \%$ (42 to 99$)$ \\
\hline Total & $97(66 \%)$ & $\begin{array}{l}13 \\
(9 \%)\end{array}$ & $\begin{array}{l}8 \\
(5 \%)\end{array}$ & $\begin{array}{l}30 \\
(20 \%)\end{array}$ & 148 & 106 & $72 \%$ (61 to 80$)$ \\
\hline \multicolumn{8}{|c|}{ Adjudication committee probability } \\
\hline & $\begin{array}{l}81 \\
(55 \%)\end{array}$ & $\begin{array}{l}15 \\
(10 \%)\end{array}$ & $\begin{array}{l}13 \\
(9 \%)\end{array}$ & $\begin{array}{l}39 \\
(26 \%)\end{array}$ & & & \\
\hline
\end{tabular}

CAP, community-acquired pneumonia; LUS, lung ultrasound. 
Table 3 Antibiotic prescription according to final diagnosis in the 47 patients with treatment changes after LUS

\begin{tabular}{|c|c|c|c|c|}
\hline & \multicolumn{4}{|c|}{ Post LUS CAP probabilities } \\
\hline & Definite & Probable & Possible & Excluded \\
\hline Antibiotic treatment withdrawn & 0 & 3 & 3 & 20 \\
\hline
\end{tabular}

CAP, community-acquired pneumonia; LUS, lung ultrasound.

very good LRs with a much higher diagnostic certainty of CAP with LUS. Nevertheless, Reissig et $a t^{22}$ estimated that $8 \%$ of CAP was not detectable with LUS. Another point-of-care ultrasound approach involving lung, heart and deep veins also showed promising results. ${ }^{23}$

In our study, we applied LUS criteria for pneumonia described in a seminal study and confirmed in a consensus conference. ${ }^{1415}$ They included direct visualisation of a condensation or signs of a localised pulmonary oedema (B lines $>3$ by spot). We chose a modified eightpoint method by adding up and down posterior points. ${ }^{15}$ A recent study demonstrated that the 8-point method had similar performances for acute heart failure diagnosis that the 28-point one, ${ }^{24}$ and was less time consuming. ${ }^{19}$ When LUS was performed by experienced physicians, the procedure time can vary from less than 5 min in Cortellaro $e t a l$ s study ${ }^{19}$ to $7-13$ min in Testa $e$ t al's study. ${ }^{25}$ In our study, the mean time to perform LUS was 6 min, whereas this procedure was not only performed by experts but also by beginners. We added posterior points since pneumonia regularly occurred in posterior parts of the lung. Furthermore, CXR anteroposterior view performed in bedridden patient can increase the risk of falsely negative CXR. $^{26}$

LUS versus CXR performances were compared in several studies, ${ }^{18} 19$ and in two meta-analysis. ${ }^{16}{ }^{26}$ Overall, LUS sensitivity was higher than CXR, 0.95 (95\% CI 0.93 to 0.97 ) versus 0.77 (95\% CI 0.73 to 0.80 ), respectively, while specificity was not significantly different. ${ }^{26}$ Furthermore, in a geriatric population, almost half of our population, LUS exhibited higher diagnostic accuracy for pneumonia than CXR, 0.90 (95\% CI 0.83 to 0.96 ) versus 0.67 (95\% CI 0.60 to 0.74$)$, respectively. ${ }^{23}$ In a study using

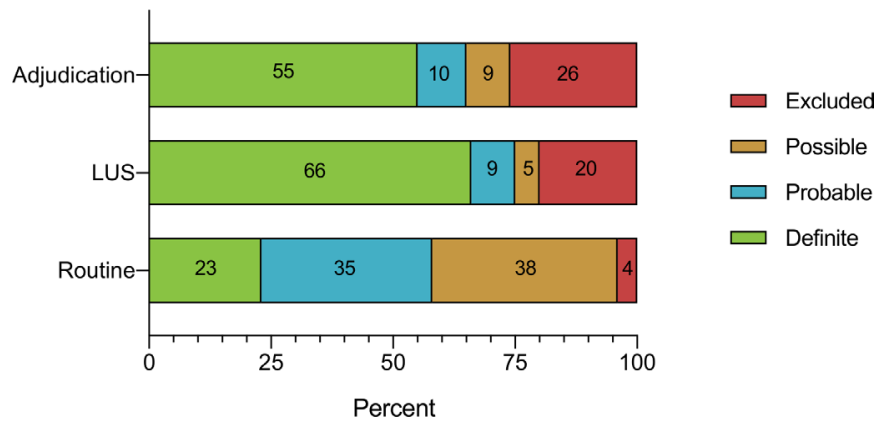

Figure 3 CAP diagnosis concordance between routine diagnostic procedure, LUS examination and adjudication committee. CAP, community-acquired pneumonia; LUS, lung ultrasound.
CT scan as gold standard, LUS sensitivity and specificity to diagnose CAP were about $90 \%$ and $97 \%$, respectively. ${ }^{27}$ Overall, LUS has showed good diagnostic performances even when compared with CT scan in the literature.

Our study was a preliminary investigation, which aimed to assess diagnostic and therapeutic changes in CAP following LUS, as it was previously reported for CT scan. ${ }^{12}$ Unlike CT, ultrasound devices are widely available in ED, thus LUS could be increasingly used for the CAP diagnosis. LUS is performed at the bedside in few minutes without any irradiation. Importantly, our findings were not different from the Claessens et al's study, ${ }^{12}$ with CAP diagnosis probability changes following LUS and CT scan in $72 \%$ versus $59 \%$, respectively. Moreover, in our study, $77 \%$ of the CAP diagnosis probabilities following LUS were in accordance with the adjudication committee compared with $80 \%$ following CT scan in the Claessens et als study. ${ }^{12}$ Likewise, antibiotics prescription changes following LUS and CT scan were not different, antibiotic treatment was started after LUS in 54\% and $45 \%$ after CT scan, and discontinued in $22 \%$ versus $14 \%$, respectively.

\section{Limitations}

Our study has several limitations. First, the CXR was interpreted by the patient's treating emergency physician and not by a radiologist, which may have led to lower diagnostic performance. However, this is the routine procedure in many EDs. Second, as this was a pragmatic study, diagnostic testing was at the discretion of the treating physician, and CT scan was not required for CAP diagnosis, which may have led to missing CAP diagnosis in the ED. However, to date, a CT scan is not recommended as a routine radiological test for the diagnosis of CAP in the ED. Moreover, the independent experts involved in the adjudication committee had access to the detailed medical records of the included patients. Third, some potentially eligible patients were missed when a local investigator was not present and during ED's busy periods, which may have led to selection bias. Fourth, the study required the performing of LUS after the routine diagnosis procedure, which may have led to a selection of patients with less severe disease. Fifth, the adjudication committee experts had access to the LUS examination results and may have been influenced by LUS findings. Sixth, the prevalence of CAP in our study was high (about $80 \%$ ), and our results should be interpreted with caution in other sites with lower prevalence of the disease. Finally, some variables were not collected in our study, such as 
information on residence in nursing homes or nursing facilities, which limits the description of our population and, therefore, the extrapolation of our results, as well as the information of overlapping diagnoses in subjects with CAP (eg, CAP and heart failure).

\section{CONCLUSION}

In conclusion, our study suggests that adding LUS to the routine diagnosis procedure could improve CAP diagnosis accuracy and could help to reduce diagnosis uncertainty and unnecessary antibiotic prescriptions. Other studies are warranted to compare LUS to CT scan in CAP diagnosis in the ED.

\section{Author affiliations}

${ }^{1}$ Emergency Department, Centre Hospitalier Universitaire de Nantes, Nantes, France ${ }^{2}$ Department of Emergency Medicine, Faculte de Medicine, Universite de Nantes, Nantes, France

${ }^{3}$ Department of Emergency Medicine, CHU de Poitiers, Poitiers, France ${ }^{4}$ Department of Infectious Diseases, Faculte de Medicine, University Hospital of Nantes and CIC 1413, INSERM, Nantes, France

${ }^{5}$ Department of Emergency Medicine, Hospital Center Saint Nazaire, Saint Nazaire, France

${ }^{6}$ Department of Emergency Medicine, CHD Vendée, La Roche-sur-Yon, France

\section{Twitter Philippe Le Conte @PhilippeLeConte}

Contributors EM and PLC conceived the study and wrote the manuscript. FJ, HdC and BG were part of the adjudication committee. NM, QLB, EB and DH were the local investigators. All authors read and approved the final manuscript. PLC is the guarantor of this paper.

Funding The authors have not declared a specific grant for this research from any funding agency in the public, commercial or not-for-profit sectors.

Competing interests None declared.

Patient and public involvement Patients and/or the public were not involved in the design, or conduct, or reporting, or dissemination plans of this research.

Patient consent for publication Not applicable.

Ethics approval The Nantes University Hospital's ethical committee approved the study protocol and informed consent procedures (Groupe Nantais d'Éthique, RC16_0439). All patients provided written informed consent before inclusion in the study.

Provenance and peer review Not commissioned; externally peer reviewed.

Data availability statement Data are available upon reasonable request. The datasets used and/or analysed during the current study are available from the corresponding author on reasonable request.

Open access This is an open access article distributed in accordance with the Creative Commons Attribution Non Commercial (CC BY-NC 4.0) license, which permits others to distribute, remix, adapt, build upon this work non-commercially, and license their derivative works on different terms, provided the original work is properly cited, appropriate credit is given, any changes made indicated, and the use is non-commercial. See: http://creativecommons.org/licenses/by-nc/4.0/.

\section{ORCID iDs}

Hugo de Carvalho http://orcid.org/0000-0003-4101-695X

Emmanuel Montassier http://orcid.org/0000-0002-2313-1172

Philippe Le Conte http://orcid.org/0000-0002-3309-6112

\section{REFERENCES}

1 Wunderink RG, Waterer G. Advances in the causes and management of community acquired pneumonia in adults. BMJ 2017;358:j2471.

2 Xu J, Murphy SL, Kochanek KD. Deaths: final data for 2016. Nat/ Vital Stat Rep 2018;67:1-76.
3 Houck PM, Bratzler DW, Nsa W, et al. Timing of antibiotic administration and outcomes for Medicare patients hospitalized with community-acquired pneumonia. Arch Intern Med 2004;164:637-44.

4 Mandell LA, Wunderink RG, Anzueto A, et al. Infectious diseases Society of America/American thoracic Society consensus guidelines on the management of community-acquired pneumonia in adults. Clin Infect Dis 2007;44 Suppl 2:S27-72.

5 Metlay JP, Fine MJ. Testing strategies in the initial management of patients with community-acquired pneumonia. Ann Intern Med 2003:138:109-18.

6 Delerme S, Ray P. Acute respiratory failure in the elderly: diagnosis and prognosis. Age Ageing 2008;37:251-7.

7 Ochoa-Gondar O, Vila-Córcoles A, de Diego C, et al. The burden of community-acquired pneumonia in the elderly: the Spanish EVAN-65 study. BMC Public Health 2008;8:222.

8 Morimoto K, Suzuki M, Ishifuji T, et al. The burden and etiology of community-onset pneumonia in the aging Japanese population: a multicenter prospective study. PLoS One 2015;10:e0122247.

9 Ray P, Birolleau S, Lefort Y, et al. Acute respiratory failure in the elderly: etiology, emergency diagnosis and prognosis. Crit Care 2006; $10:$ R82

10 Hopstaken RM, Witbraad T, van Engelshoven JMA, et al. InterObserver variation in the interpretation of chest radiographs for pneumonia in community-acquired lower respiratory tract infections. Clin Radiol 2004;59:743-52.

11 Self WH, Courtney DM, McNaughton CD, et al. High discordance of chest X-ray and computed tomography for detection of pulmonary opacities in ED patients: implications for diagnosing pneumonia. Am J Emerg Med 2013;31:401-5.

12 Claessens Y-E, Debray M-P, Tubach F, et al. Early chest computed tomography scan to assist diagnosis and guide treatment decision for suspected community-acquired pneumonia. Am J Respir Crit Care Med 2015;192:974-82.

13 Smith-Bindman R, Lipson J, Marcus R, et al. Radiation dose associated with common computed tomography examinations and the associated lifetime attributable risk of cancer. Arch Intern Med 2009;169:2078-86.

14 Lichtenstein DA, Mezière GA, Lagoueyte J-F, et al. A-lines and B-lines: lung ultrasound as a bedside tool for predicting pulmonary artery occlusion pressure in the critically ill. Chest 2009;136:1014-20.

15 Volpicelli G, Elbarbary M, Blaivas M, et al. International evidencebased recommendations for point-of-care lung ultrasound. Intensive Care Med 2012;38:577-91.

16 Llamas-Álvarez AM, Tenza-Lozano EM, Latour-Pérez J. Accuracy of lung ultrasonography in the diagnosis of pneumonia in adults: systematic review and meta-analysis. Chest 2017;151:374-82.

17 Orso D, Guglielmo N, Copetti R. Lung ultrasound in diagnosing pneumonia in the emergency department: a systematic review and meta-analysis. Eur J Emerg Med 2018;25:312.

18 Bourcier J-E, Paquet J, Seinger M, et al. Performance comparison of lung ultrasound and chest X-ray for the diagnosis of pneumonia in the ED. Am J Emerg Med 2014;32:115-8.

19 Cortellaro F, Colombo S, Coen D, et al. Lung ultrasound is an accurate diagnostic tool for the diagnosis of pneumonia in the emergency department. Emerg Med J 2012;29:19-23.

$20 \mathrm{YeX}$, Xiao H, Chen B, et al. Accuracy of lung ultrasonography versus chest radiography for the diagnosis of adult community-acquired pneumonia: review of the literature and meta-analysis. PLoS One 2015;10:e0130066.

21 Chavez MA, Shams N, Ellington LE, et al. Lung ultrasound for the diagnosis of pneumonia in adults: a systematic review and metaanalysis. Respir Res 2014;15:50.

22 Reissig A, Copetti R, Mathis G, et al. Lung ultrasound in the diagnosis and follow-up of community-acquired pneumonia: a prospective, multicenter, diagnostic accuracy study. Chest 2012;142:965-72.

23 Ticinesi A, Lauretani F, Nouvenne A, et al. Lung ultrasound and chest $\mathrm{X}$-ray for detecting pneumonia in an acute geriatric ward. Medicine 2016;95:e4153.

24 Buessler A, Chouihed T, Duarte K, et al. Accuracy of Several Lung Ultrasound Methods for the Diagnosis of Acute Heart Failure in the ED: A Multicenter Prospective Study. Chest 2020;157:99-110.

25 Testa A, Soldati G, Copetti R, et al. Early recognition of the 2009 pandemic influenza $A(\mathrm{H} 1 \mathrm{~N} 1)$ pneumonia by chest ultrasound. Crit Care 2012;16:R30.

26 Seo H, Cha S-I, Shin K-M, et al. Community-Acquired pneumonia with negative chest radiography findings: clinical and radiological features. Respiration 2019;97:508-17.

27 Nazerian P, Vanni S, Volpicelli G, et al. Accuracy of point-ofcare multiorgan ultrasonography for the diagnosis of pulmonary embolism. Chest 2014;145:950-7. 